\title{
A desnaturalização das crises globais e o conhecimento necessário em saúde
}

The denaturalization of global crises and the necessary knowledge in health La desnaturalización de las crisis globales y el conocimiento necesario en salud

Luiz Augusto Cassanha Galvão

\section{Resumo}

GALVÃO, L. A. C. A desnaturalização das crises globais e o conhecimento necessário em saúde. Rev. C\&Trópico, v. 45, n. 2, p. 11-22, 2021. DOI: https://doi.org/10.33148/cetropi$\operatorname{cov} 45 n 2(2021) \operatorname{art} 1$

Ao usar como referência a "autópsia social" da onda de calor de Chicago de 1995, o artigo examina as crises globais, particularmente as mudanças climáticas, seus quadros teóricos e as informações disponíveis para analisar o problema. Em comparação com o quadro de autópsia social, o modelo analítico atual é insuficiente para revelar os processos sociais e políticos subjacentes das crises globais. A ausência de um quadro analítico adequado desafia o estabelecimento de políticas públicas adequadas para enfrentar a atual crise global. Isso também favorece a "naturalização" dos processos, escondendo o componente político-social, que se revela como uma evidência de saúde, mas não como um processo social de longo prazo e determinante.

Palavras-chave: Mudança climática. Agenda 2030. Autópsia social. desnaturalização de desastres. Vulnerabilidade socioambiental. ODS3.

\section{Abstract}

GALVÃO, L. A. C. The denaturalization of global crises and the necessary knowledge in health. Rev. C\&Trópico, v. 45, n. 2, p. 11-22, 2021. DOI: https://doi.org/10.33148/cetropi$\operatorname{cov} 45 n 2(2021) \operatorname{art} 1$

Using as a reference the "social autopsy" of the 1995 Chicago heat wave, the article examines the global crises, particularly climate change, its theoretical frameworks, and the information available to analyze the problem. Compared with the social autopsy framework, the current standard analytical model is insufficient to reveal the global crises' underlying social and political processes. The absence of an adequate analytical framework challenges the establishment of appropriate public policies that are needed to face the current global crisis. It also favors the "naturalization" of

1 Pesquisador do Centro de Relações Internacionais em Saúde da Fundação Oswaldo Cruz (CRIS-Fiocruz); membro da Estratégia Fiocruz da Agenda 2030 e adjunct professor da Georgetown University. E-mail: luiz.galvao@fiocruz.br Orcid: https://orcid.org/0000-0002-3918-0286 
the processes, hiding the political-social component, which reveals itself as a health piece of evidence but not as a long-term and determinant social process.

Keywords: Climate change. 2030 agenda. Social autopsy. Denaturalization of disasters. Socio-environmental vulnerability. SDG3.

\section{Resumen}

GALVÃO, L. A. C. La desnaturalización de las crisis globales y el conocimiento necesario en salud. Rev. C\&Trópico, v. 45, n. 2, p. 11-22, 2021. DOI: https://doi.org/10.33148/cetropicov45n2(2021)art1

Utilizando como referencia la "autopsia social" de la ola de calor de Chicago de 1995, el artículo examina las crisis globales, particularmente el cambio climático, sus marcos teóricos y la información disponible para analizar el problema. En comparación con el marco de la autopsia social, el modelo analítico actual es insuficiente para revelar los procesos sociales y políticos subyacentes de las crisis globales. La ausencia de un marco analítico adecuado desafía el establecimiento de políticas públicas adecuadas para abordar la actual crisis mundial. Esto también favorece la "naturalización" de los procesos, ocultando el componente político-social, que se revela como una evidencia de salud, pero no como un proceso social a largo plazo $y$ determinante.

Palabras clave: Cambio climático. Agenda 2030. Autopsia social. desnaturalización de desastres. Vulnerabilidad socioambiental. ODS3. 


\section{Introdução}

Talvez não exista um melhor exemplo sobre a desnaturalização dos desastres do que o caso do clima. Há muito tempo, verificam-se vários registros de catástrofes que dizimaram populações, cidades, estados e civilizações e foram considerados "desastres naturais". A “autópsia social” do evento de onda de calor de Chicago de 1995 (KLINENBERG, 1999) revelou a intrigada relação e as revelações sobre problemas sociais subjacentes que um fenômeno climático pode revelar. Essa autópsia sem dúvida trouxe para o debate razões políticas. O modelo utilizado naquela análise desnaturalizou o desastre, superando as categorias de bom senso e trazendo um novo enfoque e uma nova abordagem para análise de eventos ambientais, que revelam condições sociais invisíveis. O modelo analisa a morfologia social, política e econômica da vulnerabilidade, o papel do Estado na determinação dessa vulnerabilidade (estrutural e conjuntural) e as tendências dos jornalistas e funcionários políticos em tornar invisíveis tanto a economia política de vulnerabilidade quanto o papel do Estado nas reconstruções dos desastres que dominam a opinião pública e influenciam os estudos científicos.

Em 1995, a crise climática ainda era pouco conhecida até mesmo nos meios acadêmicos, e os estudos e evidências que descreveram as mudanças globais e suas consequências no clima, por várias razões, concentraram-se na mitigação e na adaptação com um grande foco no fenômeno natural e, semelhante aos estudos de ondas de calor (fenômeno que tende a aumentar durante crises climáticas), não consideravam o marco socioeconômico e cultural determinante da criação das vulnerabilidades relacionadas às mudanças globais.

Um bom exemplo é a tônica que permeia o primeiro relatório (90-92) do IPCC (1990) que, em vez de analisar o que acontecia política e socialmente nos países em desenvolvimento e a imensa desigualdade entre os que estavam causando e os que estavam sofrendo o problema, concentrou as análises no impacto e na falta de recursos para tomar medidas de adaptação, o que impacta nos países em desenvolvimento, muitos dos quais carecem de recursos para adaptação. Entendendo os prováveis impactos das mudanças climáticas sobre assentamento humano, energia, transporte, indústria e saúde humana, em tais países devem ser alta prioridade, juntos com o reforço da capacidade indígena, projetar e implementar estratégias para reduzir os impactos adversos da mudança climática.”

Agora, muitos anos depois, e com milhares de estudos feitos, se anuncia que o nível dessa crise climática não tem precedentes, os conhecimentos (valiosos) produzidos não se propõe e não realizam a autópsia da crise, a qual certamente, como no caso das ondas de calor de 95 em Chicago revelariam ângulos extremamente relevantes para poder gerar as transformações preconizadas para contribuir aos objetivos aspirados pela Agenda 2030 e seus objetivos sustentáveis.

Os efeitos esperados desse processo, principalmente nos países em desenvolvimento, são enormes. Mesmo sem autópsia do fenômeno, sabemos, segundo a OMS, que a crise climática causará mais de 250.000 mortes adicionais por ano entre 2030 e 
2050 (WHO, 2021). A OMS também adverte que as ações, urgentes e necessárias, para proteger as pessoas e o planeta devem ser intersetoriais, contribuir à mitigação das emissões, aumentar a resiliência e a adaptação à nova realidade irreversível.

Entre outros os efeitos na saúde incluem impactos diretos como aqueles das ondas de calor, tempestades, incêndios florestais, inundações ou secas e efeitos indiretos decorrentes dos efeitos da mudança climática nos ecossistemas e se relacionam com a distribuição de espécies vegetais e animais, quantidade e qualidade da água e dos alimentos. Também o clima pode levar a um aumento da migração, conflitos pelo acesso a recursos naturais, instabilidade política e mudanças nos determinantes econômicos, ambientais e sociais da saúde.

\section{As Informações em Saúde e sobre Crises Globais}

O Atlas conjunto da Organização Meteorológica Mundial (OMM) e a OMS inclui dados e textos explicativos de vários fenômenos climáticos com efeito em saúde (WMO; WHO, 2012). O gráfico abaixo sobre Dengue é um exemplo do modelo explicativo e que se pode observar não inclui nenhuma das dimensões propostas para a desnaturalização, como os contexto político-social e histórico. Como esse tipo de material também é usado nos desenhos acadêmicos se consolida um ciclo de produção acadêmica que consolida a ideia de naturalização dos fenômenos como se fossem gerados e mantidos por forças da natureza e controlados pelos governos e pacientes.

Figura 1: Modelo de interação de fatores não climáticos na transmissão da dengue proposto pela OMM e OMS

Modelo de interação de fatôres não climáticos na transmissão da dengue proposto pela OMM e OMS

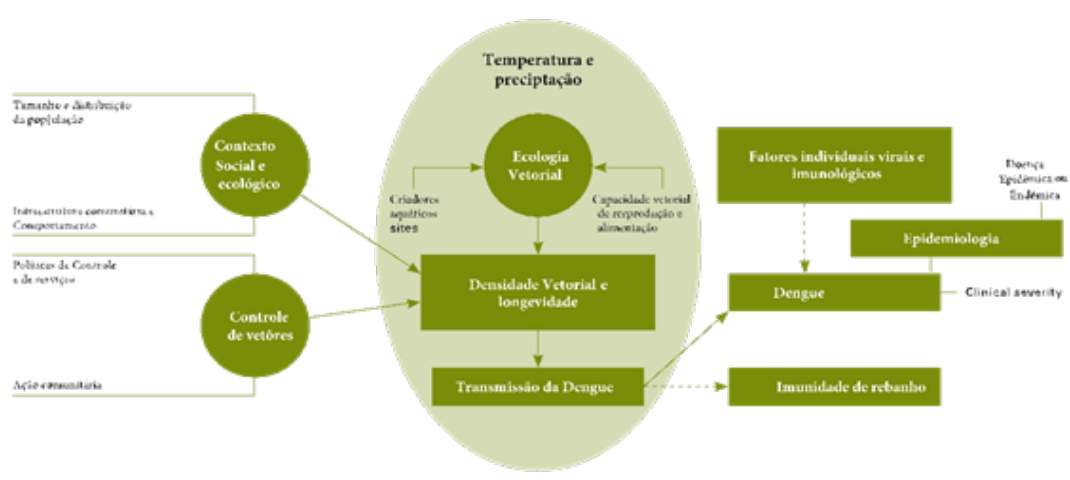

Fonte: adaptado de WMO e WHO (2012) ${ }^{2}$

2 Atlas of health and Climate. Geneva, Disponível em https://library.wmo.int/doc_num.php?explnum_ id=7790 Acesso em: 2012. $16 \mathrm{dez} 21$ 
O modelo conceitual do PNUD (2012), que foi utilizado como ponto de partida para a formulação da Agenda 2030 e de seus objetivos de desenvolvimento sustentável, tenta romper com a noção fenomenológica e determinista do desenvolvimento e inclui princípios ao centro do processo (direitos humanos, sustentabilidade e equidade) que se articulariam com processos de inclusão social e econômica, sustentabilidade e paz e segurança. Ainda que alguns dos elementos fossem considerados parte de uma desnaturalização do processo de desenvolvimento, os exemplos de articulação e ações mantêm uma visão pragmática que tende a consolidar e perpetuar a discriminação sistemática geradora de desigualdades que posteriormente são medidas por métodos cada vez mais complexos e precisos, mas que tampouco procedem com autopsiar o fenômeno e revelar as profundas raízes e processos que se escondem atrás da visão naturalizada dos eventos (Galvão, 2015).

Figura 2: Esquema sobre desenvolvimento sustentável proposto pelo PNUD/ONU, 2012

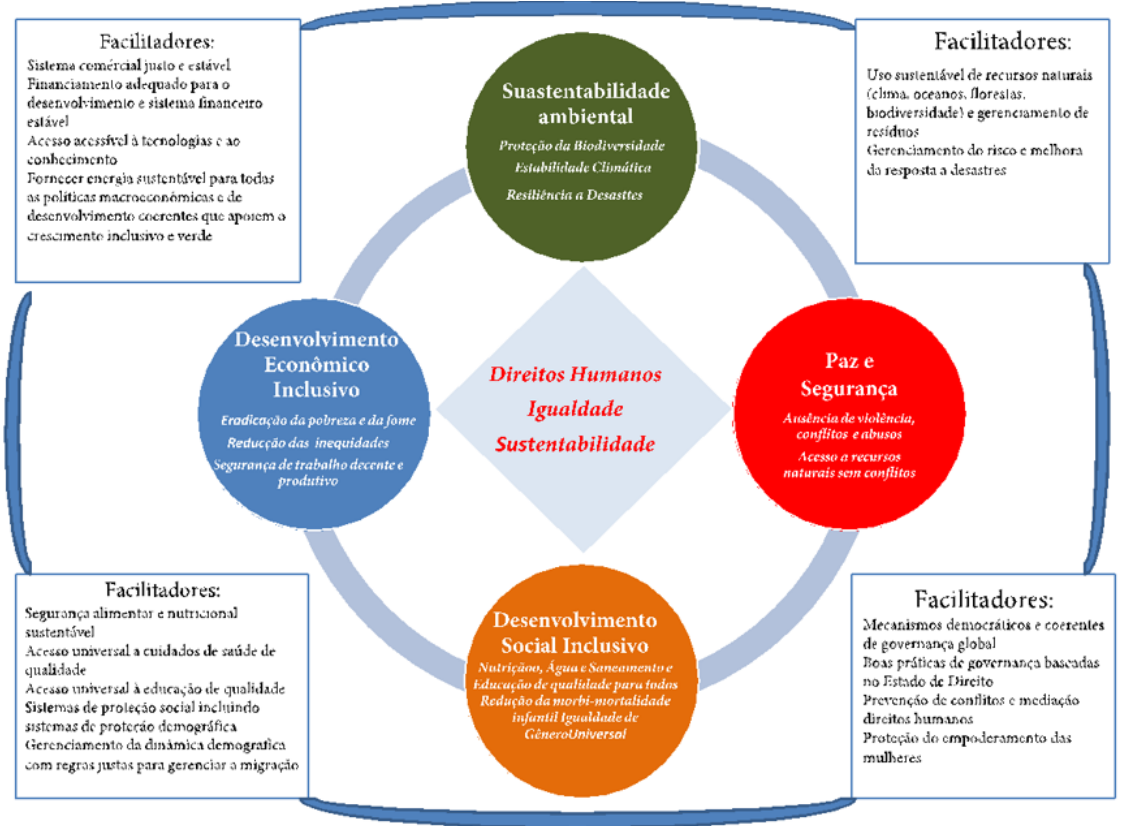

Fonte: Adaptado de PNUD (2012) $)^{3}$

3 Realizing the future we want, New York, 2012. Disponível em: https://www.un.org/millenniumgoals/pdf/ Post_2015_UNTTreport.pdf Acesso em: 14 dez. 21 
O modelo das seis transformações para o desenvolvimento sustentável, proposto pela Rede da ONU sobre Soluções para o Desenvolvimento Sustentável, trata de integrar os objetivos da Agenda 2030 explorando processos transformadores, aproximando-se mais de uma tentativa explicativa integrada e processual (SACHS et al., 2019). Ainda assim, não aprofunda aspectos fundamentais para entender questões centrais do modelo de desenvolvimento e sua dinâmica que determina as situações de vulnerabilidade e os resultados que sempre penalizam aqueles que vivem na parte puída do tecido social.

Figura 4: As seis transformações necessárias segundo os princípios de não deixar ninguém para trás, economia circular e independência da economia baseada no carvão.

\section{Não deixar ninguém para trás}
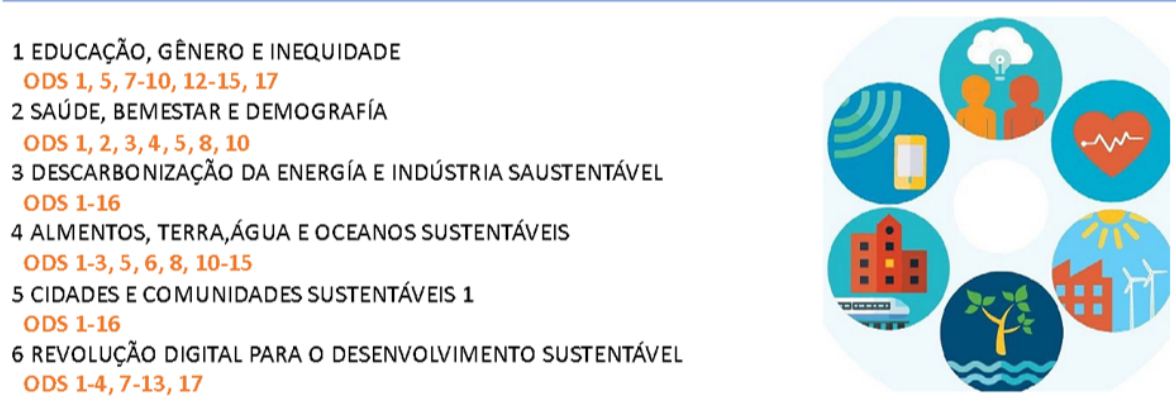

Circularidade e dissociação

Fonte: adaptado de Sachs, J.D., Schmidt-Traub, G., Mazzucato, M. et al. (2019) ${ }^{4}$.

As medições das consequências à saúde pública das mudanças globais, apesar de específicas, são feitas em uma escala que não permite assertivas sobre tendências ou mesmo avaliação de intervenções. O Instituto de Métricas de Saúde e Avaliações da Universidade de Washington (IHME) mantém uma extensa rede mundial de colaboradores e tem acesso a bases de dados que utiliza para medir os indicadores relacionados à saúde da Agenda 2030 (IHME, 2018) (Figura 5). Apesar de instrutivo e interessante, não contribui para a autópsia de desnaturalização dos fenômenos revelando a parte não natural deles. A Rede UNSDSN também mantém bases de dados para seguir os avanços na implementação da Agenda 2030 e publica um relatório anual sobre desenvolvimento sustentável e um painel com os indicadores e um índice de desenvolvimento sustentável por país (SACHS et al., 2021) (ver quadro a seguir).

4 Six Transformations to achieve the Sustainable Development Goals. Nat Sustain 2, 805-814 (2019). https://doi.org/10.1038/s41893-019-0352-9 
Figura 5: Progresso do índice de desenvolvimento sustentável
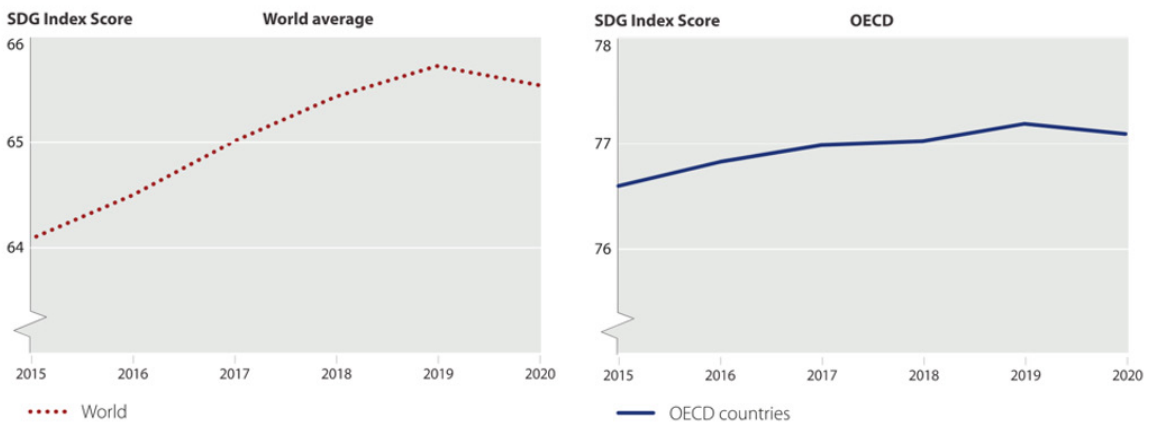

Fonte: Sachs, J., Kroll, C., Lafortune, G., Fuller, G., Woelm, F. (2021)

Figura 6: Mortalidade atribuída à contaminação do ar - exemplo das páginas do Relatório de Desenvolvimento Sustentável da UNSDSN

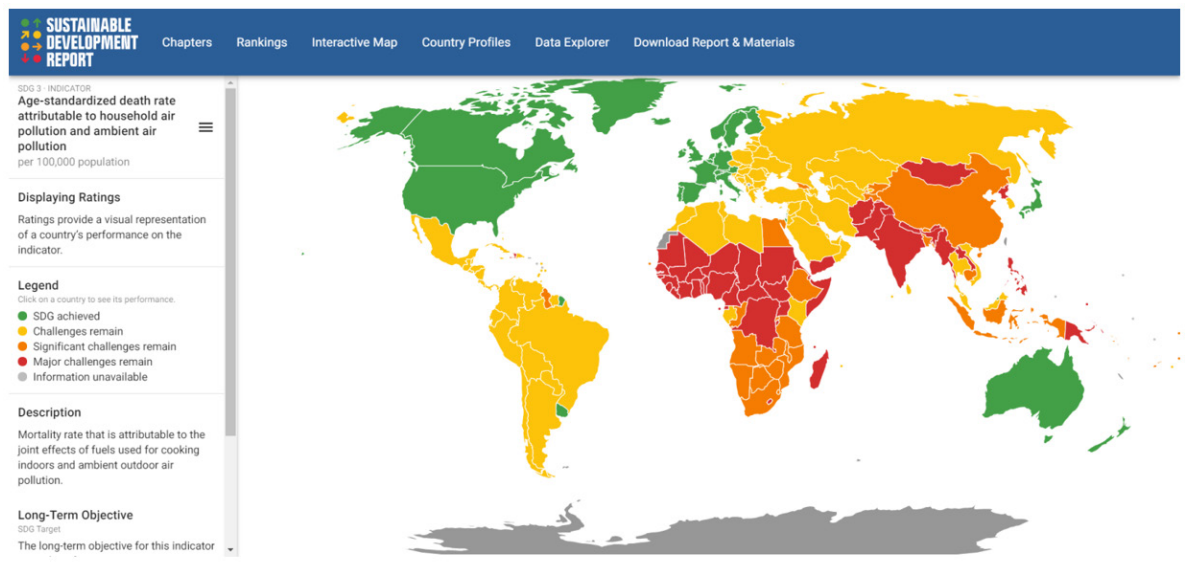

Fonte: Sachs, J., Kroll, C., Lafortune, G., Fuller, G., Woelm, F. (2021) .

5 Imagem do painel de controle dos dados do relatório. Acessível em https://dashboards.sdgindex.org/map

6 Imagem do painel de controle dos dados do relatório Disponível em https://dashboards.sdgindex.org/ map 
Figura 7: O índice de desenvolvimento sustentável do Brasil - Exemplo do índice de desenvolvimento sustentável do IHME dos indicadores relacionados à saúde

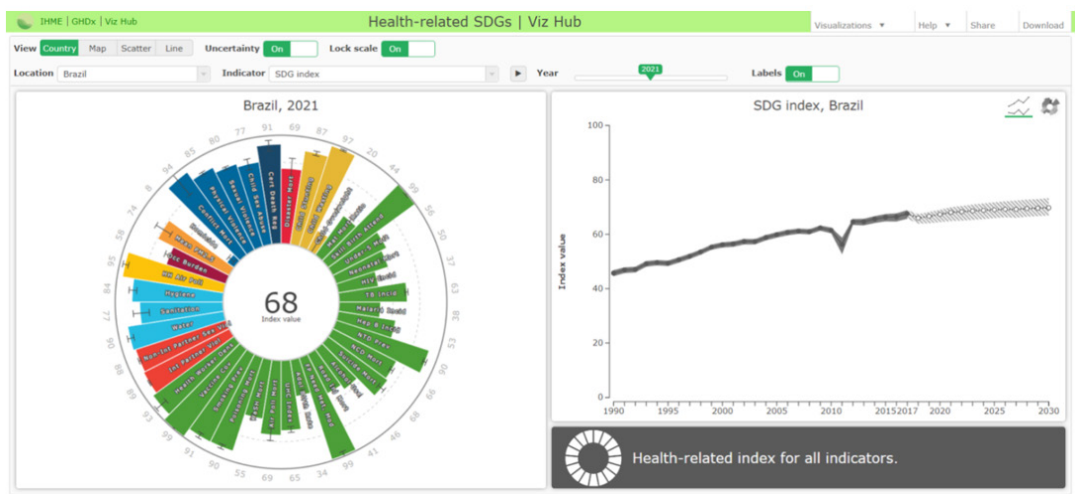

Fonte: IHME, $2018^{7}$.

O Observatório Global de Saúde da OMS (WHO, 2013), apesar de desatualizado traz algumas informações que indicam as grandes desigualdades globais em relação aos causadores do problema e aos que estão sofrendo, muitas vezes sem ajuda nem visibilidade, os problemas de saúde (Quadro 1).

Quadro 1: Disability Adjusted Life Years (Daly) Anos de vida com ajustamento pela deficiência relacionados com a mudança climática

\section{Climate change attributable DALYs per 100'000 capita}

\section{FILTERS}

\begin{tabular}{l|l} 
Last updated: 2013-06-11 & Climate change attributable DALYs per 100'000 capita \\
Indicator & 2004 \\
Location & 84.16 \\
\hline Global & 278.1 \\
Low-and-middle-income countries of the African Region & 14.66 \\
Low-and-middle-income countries of the Americas & 148.9 \\
Low-and-middle-income countries of the Eastern & 5.74 \\
Mediterranean Region & \\
Low-and-middle-income countries of the European & 139.3 \\
Region & \\
Low-and-middle-income countries of the South-East Asia & 12.36 \\
Region & \\
Low-and-middle-income countries of the Western Pacific & \\
Region &
\end{tabular}

Fonte: OMS, $2004^{8}$

7 Imagem do painel de controle da ferramenta de visualização do IHME - Institute for Health Metrics and Evaluation.

8 Imagem da página do Observatório Global de Saúde da OMS - WHO. The Global Health Observatory. 


\section{Sobre a desigualdade global na produção das informações}

Além dos problemas com o tipo de análises e dados que não permitem identificar e analisar o componente natural e o não natural das crises de saúde causadas por mudanças globais, soma-se a questão da colonização das ciências que contribui para desvios sistemáticos de desenho e interpretação de estudos (CAME et al., 2021). Também é notável a desigualdade na contribuição às publicações científicas, como nos sugere uma nota de editores, que diz que mais de mil cientistas notáveis publicaram sobre mudanças climáticas, o que mostra uma nota positiva sobre a tendência da ciência acompanhar a problemática mundial. Mas desses, apenas 122 eram mulheres, 111 do sul global, sendo que 88 eram da China e nenhum da África (SCHIPPER, 2021).

\section{4. À guisa de conclusão}

Apesar de reconhecer que existe um grande desafio científico pós-covid-19 como descreve o roteiro de pesquisa da ONU para a recuperação da Covid-19 (UN, 2021), a afirmação do secretário-geral da ONU, António Guterres de que o relatório do Grupo I do IPCC (Painel Intergovernamental sobre Mudanças Climáticas) é "um código vermelho para a humanidade" nos impele a tomar ação enquanto reconstruímos os marcos teóricos vigentes. No extenso relatório (AR6) do IPCC as principais conclusões que nos podem orientar são (IPCS, 2021):

a) Situação atual do clima: É inequívoco que a influência humana aqueceu a atmosfera, o oceano e a terra; A escala das mudanças recentes em todo o sistema não tem precedentes ao longo de muitos séculos a milhares de anos; As mudanças climáticas induzidas pelo homem já estão afetando muitos extremos climáticos em todas as regiões do mundo, que se evidenciam em ondas de calor, precipitação pesada, secas e ciclones tropicais; e o conhecimento dos processos climáticos e as evidências foram aprimorados, possibilitando uma melhor estimativa e comparação com o relatório anterior (AR5).

b) Possíveis futuros climáticos : A temperatura da superfície global continuará aumentando até pelo menos meados do século, sob todos os cenários de emissões considerados; O sistema climático se altera em razão do aumento do aquecimento global e leva a um aumento na frequência e intensidade de extremos climáticos, com ondas de calor, precipitação pesada, secas agrícolas, ciclones tropicais intensos e reduções no gelo do Mar Ártico; O aquecimento global contínuo deve intensificar ainda mais o ciclo global da água, agravando a intensidade dos eventos úmidos e secos; Com o aumento das emissões de $\mathrm{CO} 2$, os sumidouros de carbono do oceano e da terra serão menos eficazes no retardamento do acúmulo de CO2 na atmosfera; e muitas mudanças devido às emissões passadas e futuras de gases de efeito estufa são irreversíveis por séculos a milênios, especialmente mudanças no oceano, mantos de gelo e nível global do mar.

Disponível em: https://www.who.int/data/gho/data/indicators/indicator-details/GHO/climate-change-attributable-dalys-per-100000-capita 
c) Informações climáticas para avaliação de riscos e adaptação regional: Os condutores naturais "de calor" e a variabilidade interna modularão as mudanças causadas pelo homem, especialmente em escalas regionais e no curto prazo, com pouco efeito, no entanto, sobre o aquecimento global centenário; Com mais aquecimento global, cada região pode experimentar mudanças simultâneas e múltiplas; A avaliação de risco não deve descartar resultados de baixa probabilidade, como o colapso do manto de gelo, mudanças bruscas na circulação oceânica e alguns eventos extremos.

d) Limitar a mudança climática futura: Do ponto de vista da ciência física, limitar o aquecimento global induzido pelo homem a um nível específico requer limitar as emissões cumulativas de $\mathrm{CO} 2$, atingindo pelo menos emissões líquidas de $\mathrm{CO} 2$ zero, juntamente com fortes reduções em outras emissões de gases de efeito estufa. Reduções fortes, rápidas e sustentadas nas emissões de $\mathrm{CH} 4$ também limitariam o efeito de aquecimento e melhorariam a qualidade do ar; Nos cenários chamados de SSP1-1.9 e SSP1-2.6, de baixas ou muito baixas emissões de gases de efeito estufa (GEE), serão necessários anos para se obterem efeitos perceptíveis sobre as concentrações de gases de efeito estufa e aerossóis, e a qualidade do ar, em relação aos cenários de emissões de GEE elevados e muito altos (SSP3-7,0 ou SSP5-8,5). Sob esses cenários contrastantes, diferenças perceptíveis nas tendências da temperatura da superfície global começariam a emergir da variabilidade natural em cerca de 20 anos, e durante períodos mais longos para muitos outros drivers de impacto climático.

O AR6 terá ainda a contribuição do Grupo de Trabalho II que inclui um capítulo sobre saúde e do Grupo de Trabalho III, que analisará as medidas de mitigação/atenuação.

Como vemos, a ciência e as instituições continuam atuantes, mas é necessário um alto para a inovação metodológica que nos permita ir além na busca de uma compreensão desnaturalizada das crises globais e a saúde. Diferente da pandemia, a solução à ruptura social existente não poderá contar com nenhum remédio ou vacina. 


\section{Referências}

KLINENBERG, Eric. "Denaturalizing Disaster: A Social Autopsy of the 1995 Chicago Heat Wave." Theory and Society, vol. 28, no. 2, Springer, 1999, p. 239-95, http://www. jstor.org/stable/3108472.

INTERGOVERNMENTAL Panel on Climate Change. The IPCC 1990 and 1992 Assessments. Acesso em $14 \mathrm{dez} 2021 \mathrm{https} / /$ www.ipcc.ch/report/climate-change-the-ipcc-1990-and-1992-assessments/ WHO. Climate Change Acesso em 14 dez 2021 https://www.who.int/health-topics/climate-change\#tab=tab_1

WMO and WHO. Atlas of health and Climate. Geneva, 2012. Disponível e Acesso em $16 \mathrm{dez} 2021$ https://library.wmo.int/doc_num.php?explnum_id=7790

PNUD, Realizing the future we want, New York, 2012, disponível em: https://www. un.org/millenniumgoals/pdf/Post_2015_UNTTreport.pdf Acesso em 14 dez 2021Galvão, LAC. A Saúde na Era do Desenvolvimento Sustentável: análise de sua evolução e evidências. Tese de dissertação de doutorado, UFRJ/IESC. Rio de Janeiro, abril 2015, http://www.posgraduacao.iesc.ufrj.br/media/tese/1435672155.pdf

SACHS, J.D.; SCHMIDT-TRAUB; G., MAZZUCATO, M. et al. Six Transformations to achieve the Sustainable Development Goals. Nat Sustain 2, 805-814 (2019). https:// doi.org/10.1038/s41893-019-0352-9

Institute for Health Metrics and Evaluation (IHME). Health-related SDGs. Seattle, WA: IHME, University of Washington, 2018. Disponível em: http://vizhub.healthdata. org/sdg. Acesso em 15 dez 2021

Sachs, J., Kroll, C., Lafortune, G., Fuller, G., Woelm, F. (2021). The Decade of Action for the Sustainable Development Goals: Sustainable Development Report 2021. Cambridge: Cambridge University Press. DOI https://doi.org/10.1017/9781009106559

WHO. The Global Health Observatory https://www.who.int/data/gho/data/indicators/ indicator-details/GHO/climate-change-attributable-dalys-per-100000-capita. Came, H., Baker, M. \& McCreanor, T. Addressing Structural Racism Through Constitutional Transformation and Decolonization: Insights for the New Zealand Health Sector. Bioethical Inquiry 18, 59-70 (2021). https://doi.org/10.1007/s11673-020-10077-w

SCHIPPER, E. LISA F. et al. Equity in climate scholarship: a manifesto for action, Climate and Development, 13:10, 853-856, (2021) DOI: 10.1080/17565529.2021.1923308 
UN. UN Research Roadmap for the COVID-19 Recovery. New York, 2021. Disponível em: https://www.un.org/en/coronavirus/communication-resources/un-research-roadmap-covid-19-recovery. Acesso em 15 dez 2021

IPCS. AR6 Climate Change 2021: The Physical Science Basis. Disponível em: https:// www.ipcc.ch/report/ar6/wg1/ Acesso em 15 dez 2021 